\title{
The Earth as a living planet: human-type diseases in the earthquake preparation process
}

\author{
Y. F. Contoyiannis ${ }^{1}$, S. M. Potirakis ${ }^{2}$, and K. Eftaxias ${ }^{1}$ \\ ${ }^{1}$ Department of Physics, Section of Solid State Physics, University of Athens, Panepistimiopolis, 15784, Zografos, \\ Athens, Greece \\ ${ }^{2}$ Department of Electronics, Technological Education Institute (TEI) of Piraeus, 250 Thivon \& P. Ralli, 12244, Aigaleo, \\ Athens, Greece
}

Correspondence to: K. Eftaxias (ceftax@phys.uoa.gr)

Received: 17 October 2012 - Revised: 10 December 2012 - Accepted: 12 December 2012 - Published: 25 January 2013

\begin{abstract}
The new field of complex systems supports the view that a number of systems arising from disciplines as diverse as physics, biology, engineering, and economics may have certain quantitative features that are intriguingly similar. The Earth is a living planet where many complex systems run perfectly without stopping at all. The earthquake generation is a fundamental sign that the Earth is a living planet. Recently, analyses have shown that human-brain-type disease appears during the earthquake generation process. Herein, we show that human-heart-type disease appears during the earthquake preparation of the earthquake process. The investigation is mainly attempted by means of critical phenomena, which have been proposed as the likely paradigm to explain the origins of both heart electric fluctuations and fractureinduced electromagnetic fluctuations. We show that a time window of the damage evolution within the heterogeneous Earth's crust and the healthy heart's electrical action present the characteristic features of the critical point of a thermal second-order phase transition. A dramatic breakdown of critical characteristics appears in the tail of the fracture process of heterogeneous system and the injured heart's electrical action. Analyses by means of Hurst exponent and wavelet decomposition further support the hypothesis that a dynamical analogy exists between the geological and biological systems under study.
\end{abstract}

\section{Introduction}

Empirical evidence has been accumulated supporting the view that a number of systems arising from disciplines as diverse as physics, biology, engineering, and economics may have certain quantitative features that are intriguingly similar (Bar-Yam, 1997). Characteristically, de Arcangelis et al. (2006) presented evidence for universality in solar flare and earthquake (EQ) occurrence. Kossobokov et al. (2000) have explored similarities of multiple fracturing on a neutron star and on the Earth, including power-law energy distributions, clustering, and the symptoms of transition to a major rupture. Sornette and Helmstetter (2002) have presented occurrence of finite-time singularities in epidemic models of rupture, EQs, and starquakes. Abe and Suzuki (2004) have shown that the Internet shares with EQs common scale-invariant features in its temporal behaviors. Peters et al. (2002) have shown that the rain events are analogous to a variety of non-equilibrium relaxation processes in nature such as EQs and avalanches. Fukuda et al. (2003) have shown similarities between communication dynamics in the Internet and the automatic nervous system.

Importantly, strong analogies between the dynamics of geophysical and biological systems have been reported by numerous authors. Indeed, authors have proposed that dynamics of EQs and neurodynamics could be analyzed within similar mathematical frameworks (Herz and Hopfield, 1995; Rundle et al., 2002). Characteristically, driven systems of interconnected blocks with stick-slip friction capture the main features of EQ process. These models, in addition to simulating the aspects of EQs and frictional sliding, may also 
represent the dynamics of neurological networks (Herz and Hopfield, 1995, and references therein). Hopfield (1994) proposed a model for a network of $N$ integrate-and-fire neurons. In this model, the dynamical equation of $k$-th neuron (see Eq. (28) in Hopfield (1994)) is based on the HodgkinHuxley model for neurodynamics and represents the same kind of mean field limit that has been examined in connection with EQs (Rundle et al., 2002). Recently, Osorio et al. (2010) in a pioneering work under the title "Epileptic seizures: Quakes of the brain?" have shown that a dynamical analogy supported by scale-free statistics exists between epileptic seizures and EQs. More precisely, the authors performed the analysis using the following: (i) a population of different EQs, and (ii) a population of different epileptic seizures (ESs). In recent works we have shown by means of nonextensive statistical mechanics the existence of a dynamical analogy between epileptic seizures and EQs at the level of a single fault/seizure activation. Namely, we have shown that a dynamical analogy exists for the ways in which firing neurons/opening cracks organize themselves to produce a single epileptic seizure/EQ (Li et al., 2005; Kapiris et al., 2005; Eftaxias et al., 2006, 2013). This dynamical analogy by means of nonextensivity is extended to earthquakes, magnetic storms and solar flares (Balasis et al., 2011a, b).

It has been suggested that the underlying mechanism behind human heart rate regulation shares the general principles of other complex systems (Lin and Hughson, 2001 and references therein). Picoli et al. (2007) reported similarities between the dynamics of geomagnetic signals and heartbeat intervals. Herein, we investigate the existence of similarities between a geophysical pre-seismic signal and a biological signal. We focus on the dynamics of (i) fractureinduced electromagnetic (EM) radiations rooted in two well-established distinct stages of fracture of heterogeneous medium in the field, and (ii) human electrocardiogram (ECG) rooted in healthy and injured heart's electrical action.

Several techniques have been applied to detect high risk patients from ECG (Varotsos et al., 2007 and references therein). A method which not only identifies the risk but also provides an estimate of the time of an impending cardiac arrest has been recently proposed based on the analysis of electrocardiograms by means of natural time (Varotsos et al., 2007, 2011a, b). Herein, the investigation is mainly attempted by means of critical phenomena. Importantly, critical phenomena have been proposed as the likely paradigm to explain the origins of both heart rate fluctuations and fracture-induced EM fluctuations, suggesting that the theory of phase transitions and critical phenomena may be useful in elucidating the mechanism of their complex dynamics (Kiyono et al., 2004, 2005; Contoyiannis et al., 2004, 2005). One possible reason for the reported similarities concerns the way in which correlations spread thought a system comprised of subunits. Nature seems to paint the following picture: first, single isolated activated parts emerge in the system, which, subsequently, grow and multiply. This leads to cooperative effects. Long-range correlations build up through local interactions until they extend throughout the entire system. The challenge is to determine the "critical epoch" during which the "short-range" correlations evolve into "long-range" ones. Therefore, the theories of phase transitions and critical phenomena seem to be useful in elucidating similarities in different complex systems.

Characteristic features at a critical point of a second-order transition are the existence of strongly correlated fluctuations and the scale invariance in the statistical properties. More precisely, experiments and also calculations on mathematical models confirm that right at the "critical point" the subunits are well correlated even at arbitrarily large separation. Namely, the probability that a subunit is well correlated with a subunit at distance $r$ away is unity and the correlation function $C(r)$ follows long-range power-law decay. At the critical state, self-similar structures appear both in time and space. This fact is mathematically expressed through power law expressions for the distributions of spatial or temporal quantities associated with the aforementioned self-similar structures (Stanley, 1987, 1999).

Herein, analyzing human ECG time series of healthy individuals, we provide evidence that the heart dynamics include the aforementioned critical features. Moreover, we show that, beyond the appearance of a broad range of integrated selfaffine outputs and long-range correlations, the fluctuations of the healthy ECG time series are also characterized by strong anti-persistence behavior, namely, an underlying negative feedback mechanism that kicks the system far from extreme operation. The aforementioned crucial features characterize a healthy system, since such a mechanism provides adaptability, the ability to respond to various stresses and stimuli of everyday challenges (Goldberger et al., 2002). Turning focus on another complex system, fracture-induced MHz EM fields permit the real-time monitoring of the gradual damage of stressed heterogeneous materials in the Earth's crust as an earthquake is approaching, as it happens during the laboratory fracture experiments (Contoyiannis et al., 2005; Lacidogna et al., 2011). We have shown that, at some time point of the damage evolution within the Earth's crust, a critical time window emerges which possesses all the aforementioned features of critical point of a thermal second-order phase transition. During this epoch the stressed crust is in a "healthy state" for the reason that it includes critical characteristics, thus keeping the system away from extreme states.

On the other hand, below and above of the critical point of a continuous thermal phase transition, a dramatic breakdown of critical characteristics, in particular long-range correlations, appears; the correlation function turns into a rapid exponential decay (Stanley, 1987, 1999). We show that "injury" states of heart include the characteristic features of a state which is away from the critical point. Moreover, the system shows lower anti-persistent behavior. Thus, the system has lost a part of its adaptability, namely, the ability to respond to all stresses and stimuli. Importantly, a time window 
is launched in fracture-induced EM time series after the critical window appearance, which has exactly the same "injury" characteristics. The fracture has been restricted in a narrow zone completing the fracture of heterogeneous medium. We note that our finding can be supported by other studies comparing the heart dynamics of healthy people and congestive heart failure patients as well as by the heart dynamics of healthy frogs (Contoyiannis et al., 2004). The analysis of time series has been performed in terms of the recently introduced method of critical fluctuations (MCF) of thermal systems that undergo a second-order phase transition (Contoyiannis et al., 2002, 2004, 2005, 2007, 2008, 2010), Hurst exponent (Hurst, 1951; Mandelbrot and Wallis, 1968), wavelet scalogram (e.g., Pinsky, 2002; Stark, 2005; Weeks, 2007), and wavelet marginal spectrum (e.g., Yeh and Liu, 2008; Di Marco et al., 2012).

The remaining of this contribution is organized as follows. In Sect. 2 we provide concisely the necessary background knowledge on the MCF, Hurst exponent, wavelet scalogram, and wavelet marginal spectrum. In Sect. 3 we proceed to the analysis of normal vs. pathological subjects by means of MCF for both heart time series and pre-seismic EM emissions time series. In Sect. 4 we proceed to the analysis of normal vs. pathological subjects by means of Hurst exponent and wavelet decomposition. Finally, in Sect. 5 we summarize and discuss our results.

\section{Analysis methods}

In the following we present in brief the key points of the analysis methods employed in this contribution. More specifically, the method of critical fluctuations (MCF) is introduced, while the notions of Hurst exponent, $H$, and energy density of the wavelet marginal spectrum, $P_{\mathrm{d}, \Delta T}(a)$, are shortly defined.

\subsection{The method of critical fluctuations}

Recently we have introduced the method of critical fluctuations (MCF), as a method that can reveal the critical state as well as the departure from critical state. The MCF is described in detail in Contoyiannis et al. (2002). In a few words, for a given stationary time series this method estimates the distribution of waiting times $l$ in a properly defined laminar region. It is known (Schuster, 1998) that this distribution should follow power-law decay for a system possessing intermittent dynamics. On the other hand, the corresponding waiting time distribution of a conventional (noncritical dynamics) real data time series follows an exponential decay, rather than a power-law one (Contoyiannis et al., 2004), due to stochastic noise and finite size effects.

Therefore, a function that combines both power-law and exponential decay is more appropriate to fit the distribution of waiting times in a log-log scale diagram. Such a function is of the form of $\rho(x)$ :

$\rho(x)=C x^{-p_{2}} e^{-p_{3} x}$.

As a result, one can estimate the dynamics of a real time series by fitting the waiting times distribution using $\rho(x)$. The estimated values of the two characteristic exponents $p_{2}$ and $p_{3}$ reveal the underlying dynamics.

The first step of the analysis is the acquisition of the time series values histogram, in which we search, following the method of turning points (Pingel et al., 1999; Diakonos et al., 1999), for the marginally unstable fixed point, which is considered as the begin of laminar regions. According to this method, the fixed point lies in the vicinity of the abrupt variation edge of the histogram.

In a second step, we are searching for a plateau (or a region with slow variation) in the histogram. This is defined as the laminar region, which decays slowly. The end of the laminar region is treated as a free parameter determining the size of laminar region. The waiting times in the laminar region are characterized as laminar lengths $l$. We successively calculate laminar lengths and then obtain their distribution.

Finally, a fitting of the obtained distribution is performed on a $\log -\log$ plot ending up with the estimation of the $p_{2}$ and $p_{3}$ exponents. The application of the MCF method is presented step by step in the examples analyzed in Sect. 3.1.

The above described three-step procedure is repeated for other ends of the laminar region, and finally the evolution of the $p_{2}$ and $p_{3}$ exponents vs. the end of laminar region is plotted. According to the discussion in Contoyiannis et al. (2007), only values of exponent $p_{2}$ greater than 1 and values of exponent $p_{3}$ close to zero indicate proximity to critical behavior in terms of a second-order phase transition. If $p_{2}<1$, then usually $p_{3}$ is increased and a departure from criticality is signaled.

So far, the MCF has been applied on numerical experiments of thermal systems (Ising models) (Contoyiannis et al., 2002), on electromagnetic pre-seismic signals (Contoyiannis et al., 2005, 2008, 2010), and on electrocardiac signals from biological tissues (Contoyiannis et al., 2004).

Recently, we have shown that the laminar length distribution produced by a time series from ECGs of healthy frogs demonstrated power-law behavior like the corresponding distributions produced by the critical fluctuations of thermal system on the critical point of a second-order phase transition (Contoyiannis et al., 2004). We have interpreted this result as a manifestation that the healthy heart of the frog behaves as a physical system in its critical point. In other words, there is time scale invariance. Namely, the healthy heart can respond to all the temporal-scale excitations in the same way. Almost simultaneously, another team reported that the healthy human heart demonstrates characteristic features at a critical point of a second-order phase transition (Kiyono et al., 2005), 
a result similar to ours. We note that this team analyzed heartbeat time series.

\subsection{Hurst exponent}

Roughness of a geometry or a time series, or long-term memory, in a complex process is usually expressed in terms of the well-known Hurst exponent, initially introduced for the analysis of hydrological data (Hurst, 1951; Mandelbrot and Wallis, 1968) in the form of rescale range $(R / S)$ analysis.

The $R / S$ analysis is based on two quantities: first, the range $R_{n}$, which is the difference between the maximum and minimum values of the accumulated departure of the time series from the mean, calculated over each one $(n=1,2, \ldots, d)$ of the $m$-samples long sub-series in which the time series can be divided, and, second, the standard deviation of the corresponding sub-series $S_{n}$. The so-called rescaled range is exactly the ratio of $R$ by $S$. Hurst found that a $R / S$ scale by power law as time (i.e., the sample length $m$ of the sub-series) increases:

$$
(R / S)_{m} \propto m^{H},
$$

where $H$ is the Hurst exponent, an empirical relation well describing a variety of time series of natural phenomena. The exponent $H$ is estimated as the linear slope of a $\log (R / S)_{m}-\log m$ representation.

\subsection{Wavelet scalogram and wavelet marginal spectrum}

The frequency analysis of non-stationary signals has been initially addressed by the introduction of the short-time Fourier transform (STFT) and the associated time-frequency representation, which is known as a "spectrogram" (Nawab and Quatieri, 1988; Oppenheim et al., 1999). An advance on STFT is considered to be the continuous wavelet transform (CWT) (Pinsky, 2002). A detailed comparison of the STFT and the WT can be found in Kim and Kim (2001). The wavelet transform (WT) is a kind of multi-resolution analysis (analysis of the signal at different frequencies with different resolutions) and is defined as the convolution of the signal under analysis with a set of wavelet functions generated by the "mother wavelet" by dilation and translation (Daubechies, 1992; Mallat, 1998; Stark, 2005):

$$
\begin{aligned}
& W_{\psi}^{x}(a, b)= \\
& \frac{1}{\sqrt{c_{\psi}|a|}} \int_{-\infty}^{+\infty} x(t) \cdot \psi^{*}\left(\frac{t-b}{a}\right) \mathrm{d} t, a \neq 0, t \in \Re .
\end{aligned}
$$

$\psi(t)$ is the mother wavelet function, while $\psi_{a, b}(t)=$ $\frac{1}{\sqrt{|a|}} \psi\left(\frac{t-b}{a}\right)$ is the wavelet, i.e., the dilated and translated version of the mother wavelet. $a$ is called the scale or the dilatation parameter, $b$ translation or offset parameter or shift or lag, and $\frac{1}{\sqrt{c_{\psi}|a|}}$ a normalization factor, which ensures the satisfaction of the admissibility condition and the energy normalization throughout scales (Stark, 2005). Several options have been proposed for the calculation of the discretized CWT (e.g., Torrence and Compo, 1998; Stark, 2005; Rioul and Duhamel, 1992; Weeks, 2007).

The wavelet analogue to the spectrogram is the scalogram, defined as

$\operatorname{Scalogram}(x)=\left|W_{\psi}^{x}(a, b)\right|^{2}$.

Therefore, the resulting visual representation of a scalogram is a function of scale $(a)$ and time $(b)$. Usually employed mother wavelets are the Haar, the Morlet, the Mexican hat and the Meyer functions (Daubechies, 1992).

The wavelet scale has similar meaning to the scale in maps; i.e., large scale corresponds to "overall view", or long-term behavior, while small scale corresponds to "detail view", or local behavior. It could be considered corresponding to the inverse to "frequency" if one likes to refer to Fourier analysis terms. WT has proved to be an ideal tool for measuring singularities based on the decay/growth rate of wavelet transform coefficients with respect to scales (Mallat and Hwang, 1992; Li and Liner, 2005).

The scale marginal $P_{\Delta T}(a)$ of the power in the time interval $\Delta T=\left[t_{1}, t_{2}\right]$ (or marginal energy density with respect to scale over a time interval) is frequently called "the wavelet marginal spectrum" and is obtained by integration over time interval $\Delta T$ (Di Marco et al., 2012; Yeh and Liu, 2008):

$P_{\Delta T}(a)=\sum_{b=t_{1}}^{t_{2}}\left|W_{\psi}^{x}(a, b)\right|^{2}$

We investigate here the energy density of the wavelet marginal spectrum as a discrete probability distribution, $P_{\mathrm{d}, \Delta T}(a)$ :

$P_{\mathrm{d}, \Delta T}(a)=\frac{P_{\Delta T}(a)}{\sum_{a} P_{\Delta T}(a)}$.

\section{Normal vs. pathological subjects by means of MCF}

In this section we study the existence of similarities in the breakdown that comes with the transition from the critical healthy epoch to the pathological one in both the biological and the geophysical signals under study. The analysis is performed by means of the MCF method.

\subsection{Focus on the human heart dynamics}

In this sub-section we investigate the application of the MCF method on ECGs recorded on human subjects both healthy and suffering from heart infraction. The data (ECGs) were taken from the Physikalisch-Technische Bundesanstalt 
(PTB) Diagnostic ECG Database ${ }^{1}$ (Bousseljot et al., 1995; Kreiseler and Bousseljot, 1995; Goldberger et al., 2000).

In the following we present the application of the MCF method on a healthy human ECG labeled as "p121" and a representative example of human heart Myocardial infraction under the code name "p023". The step-by-step procedure is described in detail as follows:

- First, the original ECG data were pre-processed in order to ensure a minimum cumulative stationarity of the time series to be analyzed. The times-series under analysis, $V(t)$, were obtained by restricting to the lower fluctuations, i.e., clipping the high peaks of the original time series. The ECGs of "p121" (healthy) and "p023" (infraction) are shown in Fig. 1a and e, respectively. The analyzed times-series correspond to the recorded voltage values, which fall within the interval $[-0.08,0.3]$ and $[-0.2,0.3]$, correspondingly.

- A typical characteristic of the histogram of a time series obeying to critical intermittent dynamics is the existence of a plateau (or slow variation) region that decays, namely laminar region. In Fig. $1 \mathrm{~b}$ and $\mathrm{f}$ the voltagevalue distributions (histogram) of the analyzed time series are shown. The abovementioned plateau region, which usually appears in MCF, is clearly seen.

- The fixed point according to the method of turning points lies in the vicinity of the abrupt edge of the distribution (Pingel et al., 1999; Diakonos et al., 1999). From the distributions of Fig. 1b and f, the corresponding fixed points are identified to be $V=-0.06$ and $V=-0.15$, respectively. The end point, $V_{1}$, of the laminar region is treated as a free parameter, usually, inside the decay region.

- Then, the laminar lengths, $l$, are produced as the lengths that result from successive $V$ values obeying the condition $V_{\mathrm{o}} \leq V \leq V_{1}$. In others words, the laminar lengths are the waiting times inside the laminar region.

- Finally, the resulting laminar distributions $P(l)$ are plotted in log-log scale and from there the exponents $p_{2}$, $p_{3}$ are estimated by fitting the function of Eq. (1). The plots of the exponents $p_{2}, p_{3}$ vs. $V_{1}$ (the end of laminar regions) are plotted in Fig. 1c and $g$ for the healthy and the infraction case, in that order. A representative example of the corresponding laminar distributions for each one of the examined cases is given in Fig 1d and Fig. 1h, considering $V_{1}=-0.023$ for the healthy case and $V_{1}=-0.1$ for the infraction case. The continuous lines in these plots represent the fitted functions of the form of Eq. (1). The estimated values of the involved exponents are $p_{2}=1.32$ and $p_{3}=0.03$ for the healthy

\footnotetext{
${ }^{1}$ http://physionet.ph.biu.ac.il/physiobank/database/ptbdb/
}

case, while $p_{2}=0.5$ and $p_{3}=0.13$ for the pathological case.

As it can be seen from Fig. 1c, all the estimated values for the exponents $p_{2}$ and $p_{3}$ obey the condition of criticality $\left(p_{2}>1, p_{3} \cong 0\right)$ in the healthy case. These results lead us to conclude that the healthy heart is very close to a critical state in terms of second-order phase transition. Note that this is a result similar to the healthy frog's heart result (Contoyiannis et al., 2004). On the other hand, the $p_{2}, p_{3}$ exponent values shown in Fig. 1g signify that the state of the pathological case is far from critical state. The timescale invariance of the heart tissue has been lost. This means that the heart of the Myocardial infraction subject is not able to respond to the excitations of all time scales.

\subsection{Focus on earthquake dynamics through pre-seismic EM data}

During an EQ preparation process, EM emissions in the $\mathrm{MHz}$ and $\mathrm{kHz}$ bands appear one after the other a few days or a few hours prior to the occurrence of the EQ event, exactly as it happens in the laboratory (Eftaxias et al., 2001, 2011; Kapiris et al., 2004; Contoyiannis et al., 2004, 2005; Karamanos et al., 2006; Papadimitriou et al., 2008; Potirakis et al., 2012a, $\mathrm{b}, \mathrm{c})$. The first emerging $\mathrm{MHz}$ EM activity is organized during the fracture of heterogeneous medium surrounding the backbone of large and strong asperities distributed along the fault sustaining the system (Contoyiannis et al., 2005). The finally emerging $\mathrm{kHz}$ EM anomaly is thought to be due to the fracture of family of the asperities themselves (Eftaxias et al., 2001; Contoyiannis et al., 2004, 2005; Potirakis et al., 2012b; Minadakis et al., 2012).

The MCF has been applied in a number of cases on preseismic EM emissions of the $\mathrm{MHz}$ band (Contoyiannis et al., 2005, 2008, 2010). The corresponding MHz EM recordings present intervals on which the application of MCF reveals a critical behavior. These time intervals are called critical windows (CWs), and their organization is accomplished in terms of the critical point of a second-order phase transition (Contoyiannis et al., 2005).

In the following, the sequence of critical-noncritical windows in $\mathrm{MHz}$ EM time series is presented through two examples, which refer to two strong EQ events.

The first example concerns the Kozani-Grevena EQ, which occurred on 13 May 1995 in northern Greece with a magnitude of 6.5. A critical window, recorded $11 \mathrm{~h}$ before the occurrence of the EQ event, is shown in Fig. 2a. This window has duration of about $6.4 \mathrm{~h}$. The recorded amplitude $(\phi$ values) distribution of the $\mathrm{CW}$ is shown in Fig. 2b. From this distribution a fixed point $\phi_{\mathrm{o}}$ of about $385 \mathrm{mV}$ results. Following the MCF procedure the exponents $p_{2}, p_{3}$, were estimated for various exit points $\phi_{1}$. The exponents $p_{2}, p_{3}$ vs. $\phi_{1}$ are shown in Fig. 2c. As it can be observed, all the exponent values obey to critical condition $\left(p_{2}>1, p_{3} \cong 0\right)$ in this "healthy" case. Therefore, the time series of Fig. $2 \mathrm{a}$ is indeed 


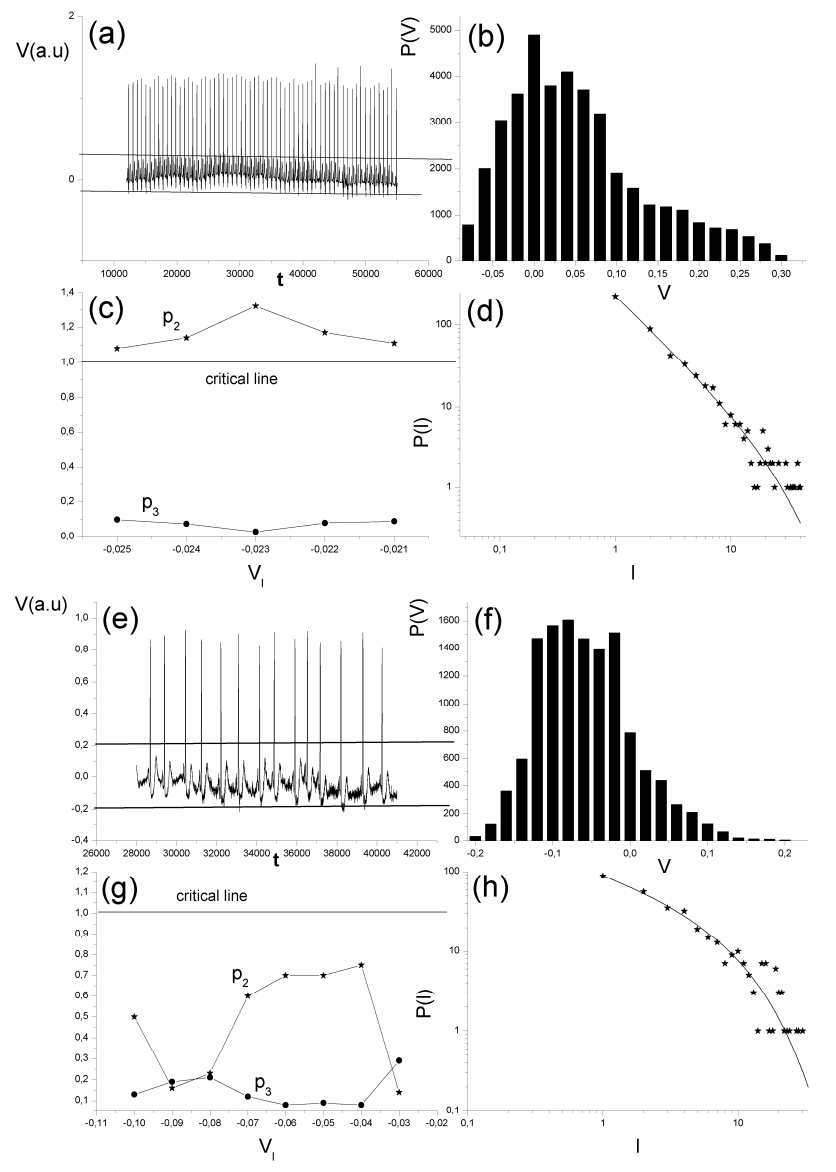

Fig. 1. (a) The ECG of the healthy subject "p121". The analyzed times-series corresponds to the recorded voltage values, which fall within the interval $[-0.08,0.3]$. (b) The voltage-value distribution of the time series of (a). From this distribution, the fixed point has been determined as $V=-0.06$. (c) The "healthy" exponents $p_{2}$, $p_{3}$ vs. $V_{1}$ (the end of laminar regions). (d) A representative example of a "healthy" laminar distribution for $V_{1}=-0.023$. (e) The ECG for a life-threatening condition of the subject "p023". The analyzed times-series corresponds to the recorded voltage values, which fall within the interval $[-0.2,0.3]$. (f) The voltage-value distribution of the time series of (e). From this distribution, the fixed point has been determined as $V=-0.15$. (g) The "pathological" exponents $p_{2}, p_{3}$ vs. $V_{1}$ are shown. (h) A representative example of a "pathological" laminar distribution for $V_{1}=-0.1$.

a critical window. A representative example of the Eq. (1) fitting for $\phi_{1}=220$ is shown in Fig. 2d. The continuous line corresponds to the fitted function of the form of Eq. (1). The estimated values of the involved exponents in this example are $p_{2}=1.21$ and $p_{3}=0.02$.

Shortly after the $\mathrm{CW}, \sim 2.5 \mathrm{~h}$ later, a clearly noncritical window appeared. The specific noncritical excerpt of the recorded EM time series, following shortly after the $\mathrm{CW}$ of Fig. 2a, almost $5.5 \mathrm{~h}$ long, is presented in Fig. 2e. The distribution of $\phi$ values is demonstrated in Fig. 2f. The
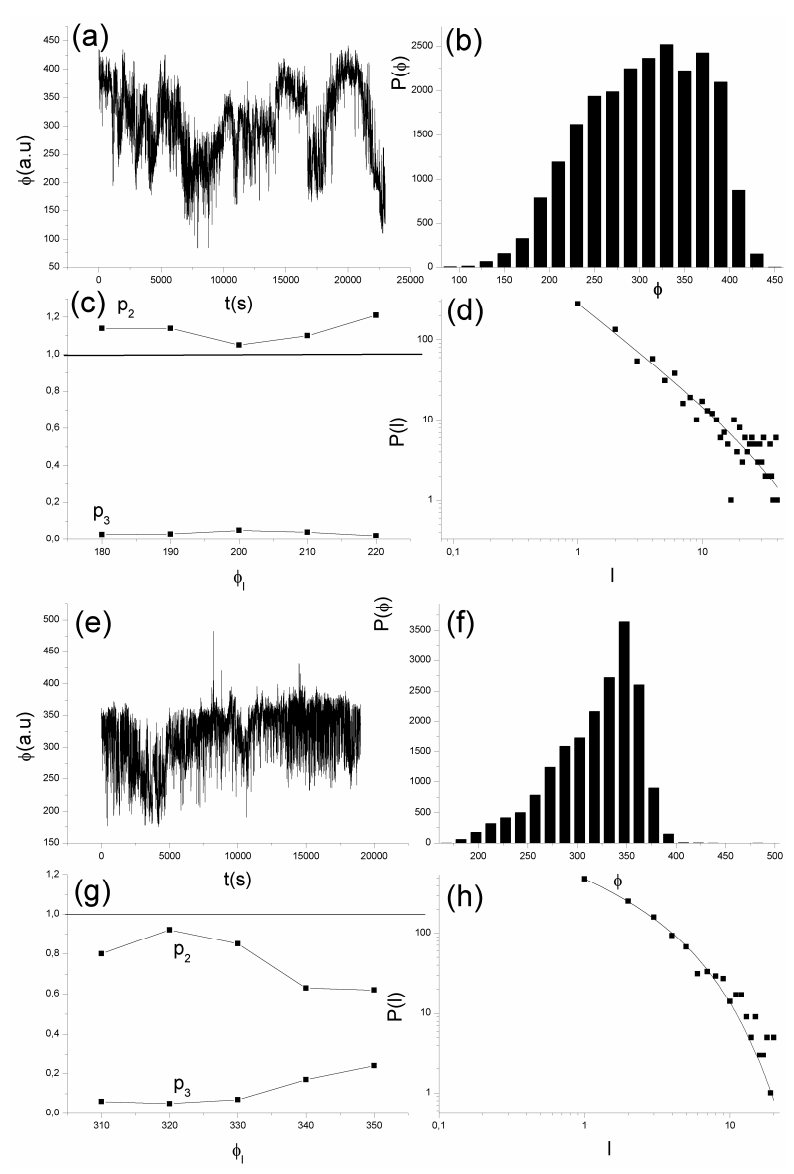

Fig. 2. (a) The CW almost $6.4 \mathrm{~h}$ long associated with the KozaniGrevena EQ (b) The histogram of the $\phi$ values of (a). (c) The corresponding "healthy" exponents $p_{2}, p_{3}$ vs. the end of laminar region $\phi_{1}$. The critical limit $\left(p_{2}=1\right)$ is also depicted. (d) A representative example of a laminar distribution of the $\mathrm{CW}$ for $\phi_{1}=220$. (e) A noncritical excerpt of the recorded EM time series, following shortly after the CW of (a), almost $5.5 \mathrm{~h}$ long. (f) The histogram of $\phi$ values of (e). (g) The "pathological" exponents $p_{2}, p_{3}$ vs. the end of laminar region $\phi_{1}$. The critical limit $\left(p_{2}=1\right)$ is also depicted. (h) A representative example of a laminar distribution of the noncritical excerpt for $\phi_{1}=350$. The departure from critical state is clear.

corresponding fixed point is identified to be $\phi_{0}=380$. Figure $2 \mathrm{~g}\left(p_{2}, p_{3}\right.$ vs. $\left.\phi_{1}\right)$ indicates that the specific time series corresponds to a state far from critical, a "pathological" heart analogous. A representative example of MCF analysis is shown in Fig. 2h. In this example, the associated exponents were estimated to be $p_{2}=0.62$ and $p_{3}=0.24$ for the exit point $\phi_{1}=350$. A few hours after the specific sequence of critical-noncritical window, the strong $(M=6.5)$ EQ event took place.

The second example refers to the L'Aquila EQ, which occurred in Central Italy on 6 April $2009(M=6.3)$. MHz EM emissions recorded prior to the specific EQ have already been presented (Contoyiannis et al., 2010). However, 
different excerpts were analyzed. Herein, a sequence of critical-noncritical window recorded almost 3 days before the EQ event is presented for the first time.

A critical window of the $\mathrm{MHz}$ recordings associated with the L'Aquila EQ is shown in Fig. 3a. This window has duration of about $2.7 \mathrm{~h}$. The corresponding amplitude ( $\phi$-values) distribution of the CW is shown in Fig. 3b. Due to the fact that this distribution is symmetric, one can select whatever edge as the fixed point. Following the MCF procedure the $p_{2}$ and $p_{3}$ exponent values were estimated, for various exit points $\phi_{1}$; the $p_{2}, p_{3}$ exponent values vs. $\phi_{1}$ are shown in Fig. 3c. It is observed that all the exponent values obey the critical condition $\left(p_{2}>1, p_{3} \cong 0\right)$. Therefore, the time series of Fig. 3a could be characterized as a critical window. A representative example of the Eq. (1) fitting for $\phi_{1}=780$ is shown in Fig. 3d. The continuous line corresponds to the fitted function of the form of Eq. (1). The estimated values of the involved exponents in this example are $p_{2}=1.18$ and $p_{3}=0.026$.

Shortly after the $\mathrm{CW}, \sim 2.7 \mathrm{~h}$ later, a clearly noncritical window appeared. The specific noncritical excerpt of the recorded EM time series, following shortly after the $\mathrm{CW}$ of Fig. 3a, almost $2.7 \mathrm{~h}$ long, is presented in Fig. 3e. The distribution of $\phi$ values is demonstrated in Fig. 3f. The corresponding fixed point is identified to be $\phi_{\mathrm{o}}=600$. Figure $3 \mathrm{~g}$ $\left(p_{2}, p_{3}\right.$ vs. $\left.\phi_{1}\right)$ indicates that the specific time series corresponds to a state far from critical, a "pathological" heart analogous. A representative example of MCF analysis is shown in Fig. 3h. In this example, the associated exponents were estimated to be $p_{2}=0.78$ and $p_{3}=0.14$ for the exit point $\phi_{1}=690$.

We observe that the MCF reveals that the dynamics of critical/noncritical window of EM fracture-induced activity is similar with that of healthy/injured heart's electrical action, correspondingly. Notice that we have shown that the noncritical window in the fracture-induced EM activity is emerged after the completeness of the "symmetry breaking" phenomenon (Contoyiannis et al., 2005), as it is predicted by criticality. This phenomenon refers to the transition from a state where the whole network is activated, characterized by high ramification, to a state where the activity is restricted to a spatially confined area. There is no direct temporal sequence between the healthy ("p121") and the pathological ("p023") ECGs, since they refer to two different subjects. However, the notion that the symmetry-breaking-type transition of a second-order phase transition is a common framework of fracture dynamics and the heart's electrical action cannot be excluded.

Herein we draw attention on the delectability of $\mathrm{MHz}$ fracture-induced EM emissions. We clarify that all the detected EM emissions are associated with surface EQs, which occurred on land or near coastline. We have already clarified this issue (e.g., Kapiris et al., 2003; Eftaxias et al., 2004; Karamanos et al., 2006). We briefly comment on this point focusing on the L'Aquila EQ case.
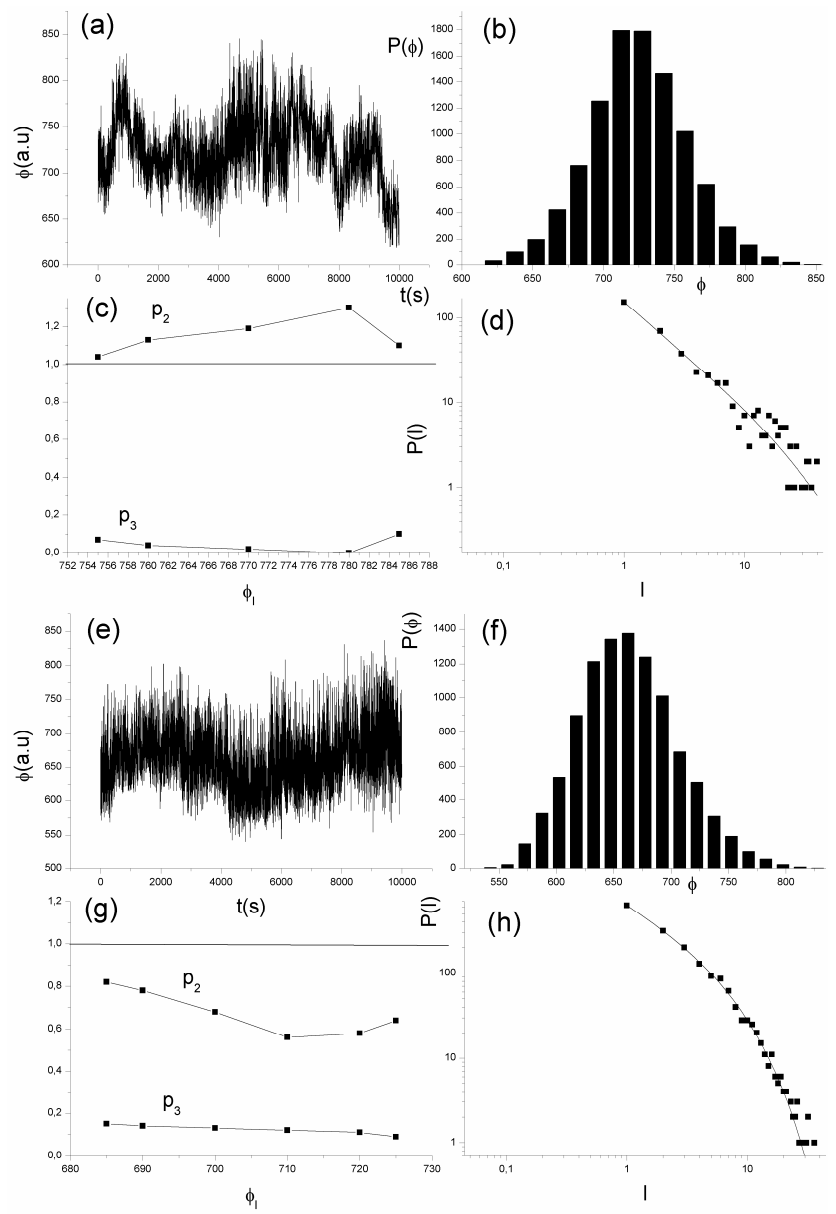

Fig. 3. (a) The CW almost $2.7 \mathrm{~h}$ long associated with the L'Aquila EQ. (b) The histogram of the $\phi$ values of (a). (c) The corresponding "healthy" exponents $p_{2}, p_{3}$ vs. the end of laminar region $\phi_{1}$. The critical limit $\left(p_{2}=1\right)$ is also depicted. (d) A representative example of a laminar distribution of the $\mathrm{CW}$ for $\phi_{1}=780$. (e) A noncritical excerpt of the recorded EM time series, following shortly after the CW of (a), almost $2.7 \mathrm{~h}$ long. (f) The histogram of $\phi$ values of (e). (g) The "pathological" exponents $p_{2}, p_{3}$ vs. the end of laminar region $\phi_{1}$. The critical limit $\left(p_{2}=1\right)$ is also depicted. (h) A representative example of a laminar distribution of the noncritical excerpt for $\phi_{1}=690$. The departure from critical state is clear.

The L'Aquila EQ was a very shallow land-based event with magnitude 6.3 that occurred on 6 April 2009 (e.g., Eftaxias et al., 2009, 2010). Notice that various methods verify this evidence. Indeed, Walters et al. (2009), using synthetic aperture radar (SAR) interferometry and body wave seismology, constrained the L'Aquila EQ parameters. They found that the top of the activated fault was located from $3.0 \mathrm{~km}$ up to $1.5 \mathrm{~km}$ below the Earth's surface. Other methods (see Table 1 in Walters et al., 2009) determine the top of this fault even up to $0 \mathrm{~km}$ from surface. We note that this view is further verified by other precursors associated with the L'Aquila EQ. More precisely, satellite thermal infrared 
anomalies were found 7 days before the EQ (30 March) (Pergola et al., 2010). Boudjada et al. (2010) have found decrease of very low frequency transmitter (VLF) signal and chorus-whistler waves about a week before L'Aquila earthquake occurrence. A ultra-low frequency (ULF) (lithosphereatmosphere-ionosphere, LAI, coupling) EM anomaly was detected from 29 March 2009 up to 3 April 2009 (Eftaxias et al., 2010). Note that the MHz EM anomalies were recorded on 26 March 2009, 2 April 2009 (Eftaxias et al., 2010), and almost 3 days before the EQ event (the presently analyzed anomaly). All the above experimental evidence conduces to the fact that the fracture process was surficial. Therefore, there is no reason why the fracture-induced EM emissions should not be directly launched to the atmosphere without absorption.

Now we focus on the intensity of the EM emission. As it was mentioned, the precursory $\mathrm{MHz}$ EM signals are produced during the fracture of the highly heterogeneous media surrounding the fault. For an EQ of magnitude $\sim 6$, the corresponding fracture process extends to a radius of $\sim 120 \mathrm{~km}$ (Bowman et al., 1998). We note that such a large extensive process is necessary in order to observe the other, already mentioned, precursors reported within or around the same time period of the $\mathrm{MHz}$ signals emergence. Moreover, as it was mentioned, during the "critical window" the underling fracture process exhibits the features characterizing the critical point of a second-order transition. Namely, the subunits (cracking area) are well correlated even at arbitrarily large separation while showing self-similar structures both in time and space (Stanley, 1987, 1999). The fact that cracking events (EM emitters) occurred in a large region and correlated strongly enhances the concept that an efficient precursory $\mathrm{MHz}$ emission was launched into the atmosphere.

We also draw attention to the fact that a fracture/faulting process is characterized by the formation of fractal pattern. Especially, as it was said, during the critical window, selfsimilar structures appear both in time and space. Fractals are highly convoluted, irregular shapes. The path length between two points is increased by requiring that the small line elements are no longer collinear. This suggests that the number of breaking bonds in a fracture network is dramatically higher in fractal fracture process in comparison to those of Euclidean fracture process. This strongly enhances the possibility to capture these pre-seismic radiations; a network of cracking-electromagnetic emitters having a fractal distribution in space is formed during the fracture of heterogeneous medium. The idea is that an extended emitting efficient fractal EM Geo-Antenna (Eftaxias et al., 2004) should be formed as a significant surface EQ approaches, launching EM radiation in the atmosphere. A fractal tortuous structure significantly increases the radiated power density, as it increases the effective dipole moment. Recently, the research area known as "fractal electrodynamics" has been established. This term was first suggested by Jaggard (1990) to identify the newly emerging branch of research, which combines fractal geometry with Maxwell's theory of electrodynamics. The abovementioned idea has been successfully tested (Eftaxias et al., 2004). Finally we note that both the efficiently transmitting part of the Earth's surface and the receiving antennae of the experimental station were located on mountainous areas with direct sight link.

\section{Normal vs. pathological subjects by means of Hurst exponent and wavelet decomposition}

In this section we further study the existence of similarities in the breakdown that accompanies the transition from the critical (healthy) epoch to the noncritical (pathological) one in both biological and geophysical signals under study. The analysis is attempted by means of Hurst exponent and wavelet decomposition, which seem to shed more light on the physical background of the observed similar breakdown in the biological and geophysical systems under study.

\subsection{Focus on the human heart dynamics}

Physiological signals in healthy state present a temporal fractal structure (Ivanov et al., 1999). The present analysis verifies the aforementioned suggestion. This fact indicates that the ECG signal is rooted in fractal forms, which consist of self-similar subunits (and sub-sub-units, etc.) that resemble the structure of the overall object. Fractal geometry appears to govern important features of cardiac mechanical function (Goldberger et al., 2002). The self-similar structures support at least one fundamental physiologic function: the rapid and efficient transport over a complex, spatially distributed network.

If a time series is a temporal fractal, then a power-law of the form

$S(f) \propto f^{-\beta}$

is obeyed, where $S(f)$ is the power spectral density and $f$ is the frequency. The spectral scaling exponent $\beta$ is a measure of the strength of time correlations. The healthy ECG under study follows the power law $S(f) \propto f^{-\beta}$. We have proven (Contoyiannis et al., 2005) that the following relation exists between the $\beta$ and $p_{2}$ exponents:

$\beta=5-3 p_{2}$.

We note that the upper bound for the spectral scaling exponent $\beta$ is $\beta=2$ by means of the "second-order phase transition" analogy. For the healthy heart the $p_{2}$ exponent is restricted in the region 1.1-1.3 (see Fig. 1c). Based on the relation (8) we conclude that the $\beta$ exponent values lie in the region 1.1-1.7.

The resulted color-type behavior of the power spectrum density $(\beta>0)$ means that the spectrum presents more power at low frequencies than at high frequencies; the system selects to transmit more power at lower frequencies. If 
all frequencies were equally important, namely white noise, it would be $\beta=0$. Moreover, the included electric events are governed by long-range temporal correlations, namely, memory: the current value of the ECG signal is correlated not only with its most recent values but also with its long-term history in a scale-invariant, fractal way. The abovementioned features imply that the data are rooted in a mechanism that is compatible with the thermal critical point.

The exponent $\beta$ is related to another exponent, the Hurst exponent $H$, by the formula

$\beta=2 H+1$

for the $\mathrm{fBm}$ model. $H$ values can lie anywhere in the range $[0,1](1<\beta<3)$ (Heneghan and McDarby, 2000) and characterize the persistent and anti-persistent properties of the signal according to the following scheme. The range $0.5<$ $H<1$ suggests persistence behavior of the signal (superdiffusion); i.e., if the amplitude of fluctuations increases in a time interval, it is expected to continue increasing in the interval immediately following. The range $0<H<0.5$ reveals anti-persistency; i.e., if the fluctuations increase in a period, they probably will continue decreasing in the interval immediately following and vice versa. Values of $H=0.5$ or $\beta=2$ indicate no correlation between successive increments. Observation of the particular values implies a transition from anti-persistent to persistent behavior during the evolution of the underlying process.

Based on the Eq. (9), we conclude that the relaxation intervals of the healthy heart are characterized by $H$ exponents distributed in the range $[0.05,0.15]$. We note that Ivanon et al. (1999) in a pioneering work have studied the human heartbeat dynamics by means of multifractality. They found that for healthy subjects the local Hurst exponents are distributed in the range $[0.07,0.17]$ consistent with our results. The resulted $H$ exponents indicate that the fluctuations in the healthy heart dynamics exhibit strong anti-persistent behavior. The associated physical information is that the control mechanism regulating the healthy heart operation is characterized by a negative feedback mechanism that kicks the system out of extreme situations. Such a mechanism provides adaptability, the ability to respond to various stresses and stimuli of everyday challenges.

The Hurst exponent also describes the roughness of the profile of the time series. The estimated $H$ values [0.05, 0.15] indicate a profile with very high roughness. In the frame of the $\mathrm{fBm}$ model, the fractal dimension is given by the relation (Lowen and Teich, 1995; Heneghan and McDarby, 2000):

$D=2-H$

therefore, the fractal dimension of the time series is close to 2 . The $H$ and $D$ values show that the fractal structure of the network is characterized by very high ramification, which permits rapid and efficient transport of electric pulses over the complex, spatially distributed cardiac tissue network.
The abovementioned relation (8) between the $\beta$ and $p_{2}$ exponents is valid only in the critical point. Therefore, we determine the Hurst exponent of the pathological ECG in terms of the $R / S$ analysis.

Figure $4 \mathrm{a}$ and $\mathrm{b}$ show that the pathological heart is characterized by $H=0.387 \pm 0.044$. The control mechanism regulating the heart dynamics keeps its anti-persistent character. However, they are less anti-persistent than in the healthy dynamics, as the scaling exponent is closer to $H=0.5$. Its ability to kick the system far from the extreme has been reduced. This result is consistent with that obtained by Ivanov et al. (1999). The profile of pathological ECG is smoother in comparison with the healthy one as it is described by a smaller/larger $H$ exponent/fractal dimension. This indicates that the network shows fewer ramifications in its structure. The capability of rapid and efficient transport over the complex, spatially distributed network has been reduced.

\subsection{Focus on the fracture dynamics}

As it has been already indicated, if a time series is a temporal fractal, then a power-law of the form of Eq. (7) is obeyed, while the Eq. (8) describes the relation between the $\beta$ and $p_{2}$ exponents. The spectral scaling exponent $\beta$ is a measure of the strength of time correlations.

In the critical window, which is emerged during the damage of the heterogeneous material, the laminar lengths follow a power law distribution with exponent $p_{2}$ distributed in the range $[1.05,1.21]$ for the Kozani-Grevena pre-seismic EM emissions (see Fig. 2c) and $[1.04,1.30]$ for the L'Aquila preseismic EM emissions (see Fig. 3c). The power spectral density follows the power law of Eq. (7) where the exponent $\beta$ is distributed in the ranges $[1.37,1.85]$ and $[1.10,1.88]$, respectively, as they are calculated using Eq. (8). The associated $H$ exponent takes values from 0.19 up to 0.43 , for KozaniGrevena, and from 0.05 up to 0.44 , for L'Aquila, through Eq. (9), while fractal dimension $D$ takes values in the range $[1.57,1.81]$ and $[1.56,1.95]$, respectively, as they are calculated using Eq. (10).

The abovementioned results show that the observed critical MHz EM field originated during the fracture of fractal forms. Elementary self-similar ruptures interact due to overlapping of their (scalar) stress fields. We focus on this point. Fractals are highly convoluted, irregular shapes. The path length between two points is increased by requiring that the small line elements are no longer collinear. This suggests that the number of breaking bonds is dramatically higher in fractal fracture process in comparison to those of Euclidean fracture process. The associated low value of $H$ exponent (close to zero) indicates high roughness of $\mathrm{MHz} \mathrm{EM}$ time series profile, while the high value of fractal dimension $D$ (close to 2) mirrors that the fracture network is characterized by high ramification. Consequently, a high amount of energy is consumed in risk-free fractures during the damage evolution of heterogeneous material that surrounds the backbone of 


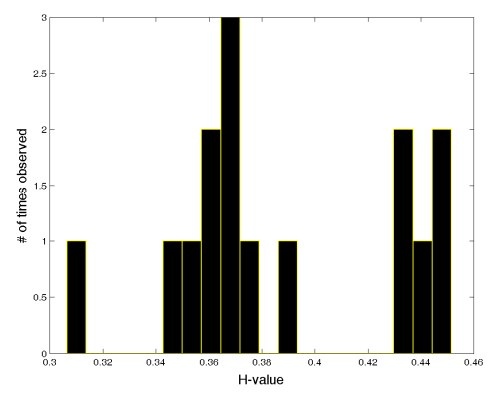

(a)
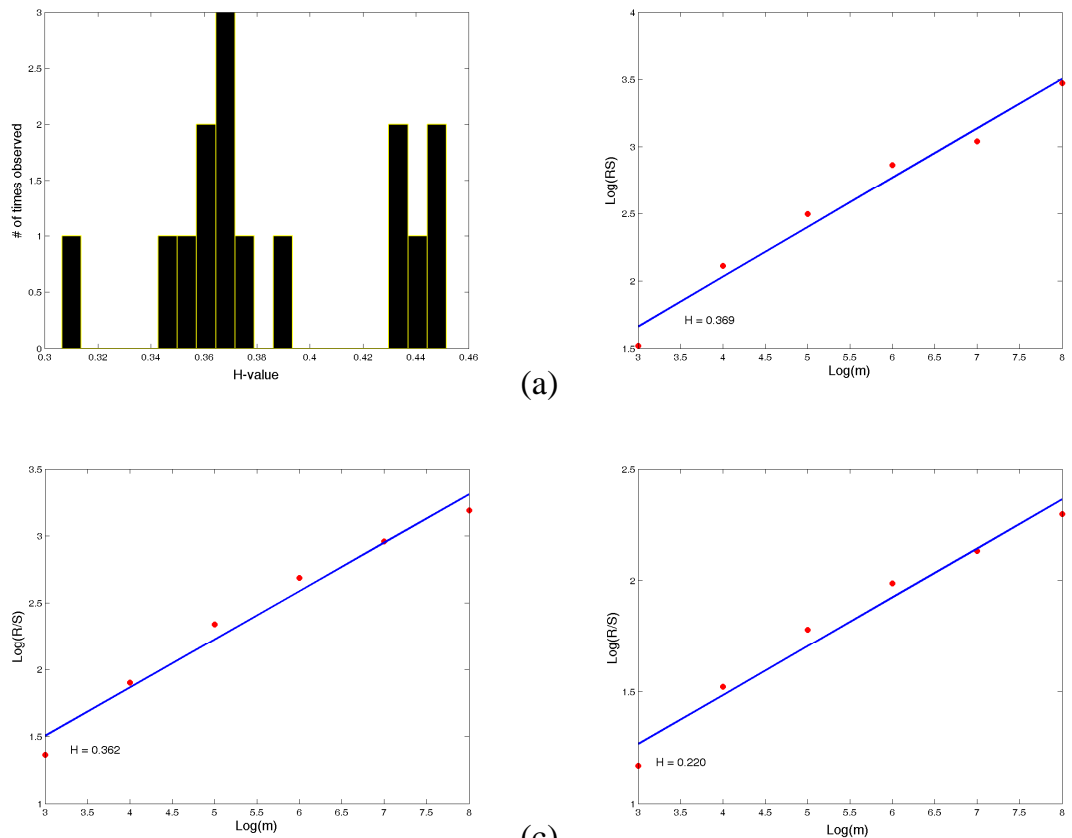

(c)

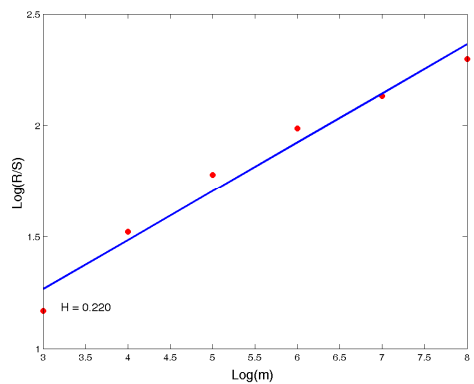

(d)

Fig. 4. (a) Histogram of the Hurst exponents resulting for the 15 relaxation parts of the "p023" ECG time series. (b) Sample application of the $R / S$ method to an indicative relaxation interval of the pathological heart taken from the ECG time series of the subject "p023". (c) $R / S$ analysis of the total length of the noncritical MHz EM emission excerpt of Fig. 2e associated with the Kozani-Grevena EQ. (d) $R / S$ analysis of the total length of the noncritical MHz EM emission excerpt of Fig. 3e associated with the L'Aquila EQ.

strong entities distributed along the fault sustaining the system. This information implies that nature plays a meaningful $1 / f$ music during the earthquake preparation process! On the other hand, the underlying mechanism is characterized by a strong negative feedback mechanism. The abovementioned mechanisms imply that the critical window includes the crucial feature of "healthy" crust function, namely, adaptability, the ability to respond to external stresses.

Figures $2 \mathrm{e}-\mathrm{h}$ and $3 \mathrm{e}-\mathrm{h}$ show that a dramatic breakdown of critical characteristics, in particular long-range correlations, appeared during the transition from the critical window to that of noncritical directional fracture; the correlation function turns into a rapid exponential decay. The fluctuations are significantly less anti-persistent than in the critical dynamics, as the scaling exponent is closer to $H=0.5$. An analysis in consecutive 1024 samples long time windows leads to the result that $H=0.351 \pm 0.044$ for the Kozani-Grevena case (see also Fig. 4c) and $H=0.218 \pm 0.028$ for the Methoni case (see also Fig. 4d). The ability of the underlying mechanism to kick the system far from of extreme has been significantly reduced. The profile of the "pathological" MHz EM time series is smoother in comparison with the critical one as it is described by a smaller/larger H exponent/fractal dimension, correspondingly. This indicates that the network shows fewer ramifications in its structure. The capability of rapid and efficient transport over the complex, spatially distributed network has been reduced.
The above presented analysis shows that significant alterations of fractal dynamics during the transition of fracture process from the "healthy" critical state to the "pathological" noncritical one are similar to those that characterize the alteration of heart dynamics when a healthy heart transitions to an injury function (Goldberger et al., 2002). A breakdown of fractal physiologic complexity accompanies the transition to the pathological state.

\subsection{Analysis by means of wavelet decomposition}

The wavelet decomposition reveals physical features that shed light on the transition from the critical to noncritical epoch of heart's operation. Figure 5a and b show a scalogram color-coded wavelet analysis of ECG time series in representative healthy and pathological relaxation windows, correspondingly. It is noted that the time series energy extends to a wider scale (or, equivalently, frequency) range, providing a more "detailed" picture, in the case of the critical epoch, i.e., in the healthy case (Fig. 5a), compared to the heart failure case (Fig. 5b). The same result is even clearly exposed through the energy density of the wavelet marginal spectrum. Indeed, Fig. 5e shows clearly that the energy content of the heart failure case is restricted to higher scales (lower frequencies) only, while the energy content of the healthy heart is distributed to a noticeably wider scale range, indicating that the healthy heart is capable of operating in a much 
more complicated way responding to many different stimuli. Taking into account that the source size is inversely proportional to the emitted frequency, we infer that the healthy heart's electrical sources are of many different sizes in contrast to the pathological case, which are expected to be limited to large sizes only. Therefore, the healthy heart system is characterized by high ramification and adaptability, results that are consistent with the results obtained through the Hurst exponent and fractal dimension analysis.

The wavelet decomposition also enhances the physical picture that emerges during the transition from critical to noncritical state of fracture. Figure $5 \mathrm{c}$ and d show a colorcoded wavelet analysis of MHz EM time series in "healthy" (critical) and "pathological" (noncritical) fracture windows, correspondingly. The critical window (Fig. 5c) and a following noncritical excerpt (Fig. 5d) of the recorded $41 \mathrm{MHz}$ electric field strength (in arbitrary units), associated with the Kozani-Grevena EQ, are respectively presented in parallel (time-aligned) to their Morlet wavelet power scalograms, with the vertical axis corresponding to the scale, $a$, of the wavelet (time scale, reciprocal to the wavelet "frequency") and the color representing the power spectral level in $\mathrm{dB}$ (side color bar). The common horizontal axis is the time (in s), denoting the relative time position from the beginning of the analyzed part of the EM recording. The brighter colors (towards red, as the color bars of Fig. 5c and d indicate) denote larger values of the wavelet power spectral level in $\mathrm{dB}$, corresponding to large fracture fluctuations. Thus, these figures show the evolution of fracture with scale and time. We concentrate on the critical "healthy" window (Fig. 5c). Coherent intense fluctuations occurred in a wide range of frequencies. The wavelet decomposition reveals a tree-like, complex, multi-scale hierarchy to the critical fracture dynamics. However, the system selects to transmit more power at lower frequencies. Higher frequency activities are rooted in small space fracture sources. In contrary, very large sources are recruited during slow fluctuations. These features indicate that the signal is rooted in well-ramified fractal forms, which are composed of self-similar subunits (and sub-sub-units, etc.) that resemble the structure of the overall object.

In contrast, Fig. 5d shows that the wavelet decomposition on fracture fluctuations from "pathological" fracture reveals the loss of complex multi-scale hierarchy. Coherent intense fluctuations occurred in a shorter range of frequencies in the noncritical window. The fracture occurs mainly on small space sources. The "healthy" tree-like network of fracture process has lost main large branching patterns. This situation is consistent with the appearance of directional activity that characterizes a state far from the critical point. The symmetry breaking has occurred.

The scalogram indicated differences between the critical and noncritical EM emissions, which are further elucidated by the corresponding energy densities of the wavelet marginal spectrums as they were calculated for both the Kozani-Grevena and the L'Aquila pre-seismic EM emissions. The results are shown in Fig. 5f and g, respectively. Like the heart case, the time series energy extends to a wider scale (or, equivalently, frequency) range in the case of the critical epoch, i.e., in the "healthy" case compared to the "pathological" (noncritical) case. It is clear that the energy content of the noncritical windows is restricted to higher scales (lower frequencies) only, while the energy content of the CWs is distributed to a noticeably wider scale range, indicating that the "healthy" fracture is characterized by a much more complicated dynamics.

In summary, accumulated evidence enables us to verify the grater complexity of the "healthy" critical dynamics compared to those of "pathological" noncritical conditions into both biological and geophysical systems, as it has already been suggested (Goldberger et al., 2002; Ivanov et al., 1999)

\section{Conclusions}

In the present work we verified the view that a number of complex systems arising from diverse disciplines may have certain quantitative features that are intriguingly similar. The performed analyses reveal the existence of strong analogies between the dynamics of fracture of heterogeneous media at geophysical scale and dynamics of heart. Critical phenomena seem to be the characteristic paradigm to explain the origins of both heart and fracture fluctuations elucidating the mechanisms of their complex dynamics. Characteristic features of a critical point of a second-order phase transition were identified in the dynamics of healthy human heart and dynamics of "critical window", which appears during the evolution of damage with time. In both systems, the activated subunits are well correlated even at arbitrarily large separation showing self-similar structures, a fact that is mathematically expressed through power law expressions for the distributions of spatial or temporal quantities associated with the aforementioned self-similar structures. Especially, the laminar lengths (waiting times) follow a powerlaw-type distribution. These results are consistent with previous ones for both systems, i.e., biological (Contoyiannis et al., 2004; Kiyono et al., 2005) and geophysical (Contoyiannis et al., 2005; Contoyiannis and Eftaxias, 2009). The fractal dimension $D$ values of the fractal structure of the networks (electrical conduction/fracture) of both systems show that they are characterized by very high ramification, which permits rapid and efficient transport of electric pulses/stresses over the complex spatially distributed network in the cardiac tissue/fractured medium, correspondingly. The dynamics of both systems also are characterized by a negative feedback mechanism that keeps the system away from extreme situations. Our analysis showed a common dramatic breakdown of the critical characteristics for the heart-failure subjects or fractured heterogeneous medium in the tail of the fracture process. The alterations refer to (i) correlations differences. The distributions of laminar lengths follow an 

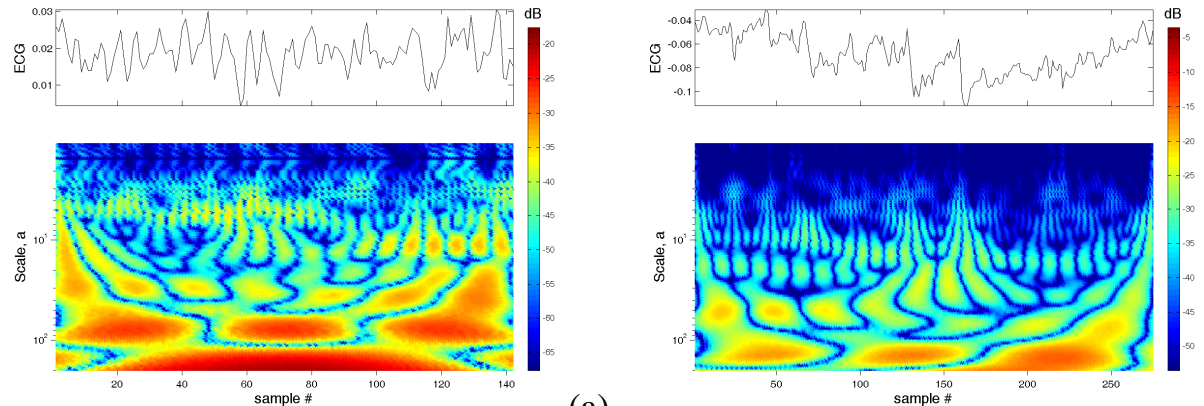

(a)

(b)

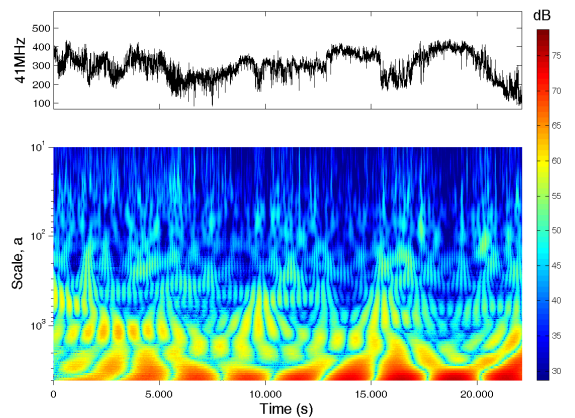

(c)

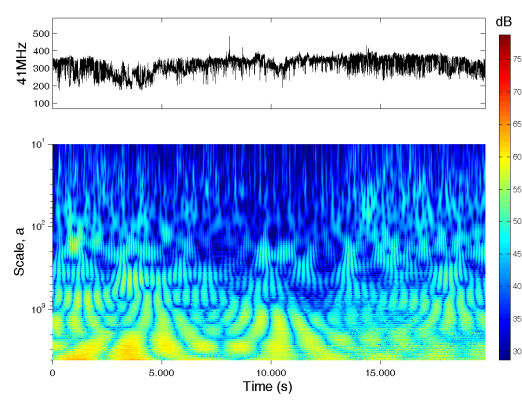

(d)


(e)

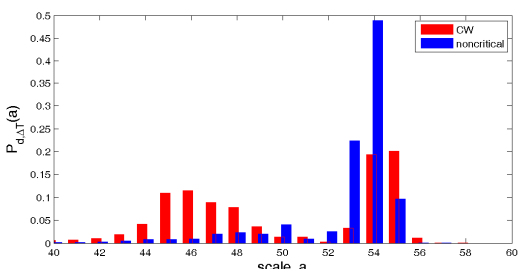

(f)
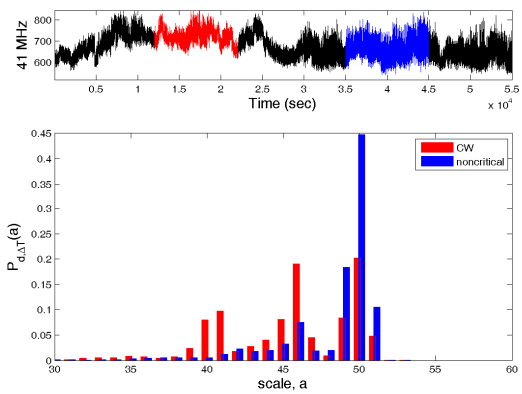

(g)

Fig. 5. (a) Morlet wavelet power scalogram (in $\mathrm{dB}$ ) of an indicative relaxation interval of the healthy heart taken from the ECG time series of the subject "p121". (b) Morlet wavelet power scalogram (in $\mathrm{dB}$ ) of an indicative relaxation interval of the pathological heart taken from the ECG time series of the subject "p023". (c) Morlet wavelet power scalogram (in dB) of the EM emissions CW associated with the KozaniGrevena EQ. (d) Morlet wavelet power scalogram (in dB) of the noncritical EM emissions associated with the Kozani-Grevena EQ. Energy density of the wavelet marginal spectrum: (e) of an indicative relaxation interval for each one of the healthy and pathological heart ECG time series, (f) of the CW and the noncritical excerpt associated with the Kozani-Grevena EQ, and (g) of the CW and the noncritical excerpt associated with the L'Aquila EQ. 
exponential-type law. (ii) The fractal dimensions have been reduced. This indicates that the fractal networks are less ramified. (iii) The electric biological and electromagnetic geophysical fluctuations are less anti-persistent compared to the healthy critical dynamics. The aforementioned found alterations are also consistent with previous studies for both systems, biological (Ivanov et al., 1999; Kiyono et al., 2005; Varotsos et al., 2007) and geophysical (Contoyiannis et al., 2005; Contoyiannis and Eftaxias, 2009). In summary, our results suggest that dynamics of heart and fracture of heterogeneous media could be analyzed within similar mathematical frameworks.

The Earth is a living planet where many complex systems run perfectly without stopping at all. Activity is constantly taking place not only on the surface, but also on the inside beneath our feet. The earthquake generation is a fundamental sign that the Earth is a living planet. The results of the present work show that human-type disease characteristics appear during the earthquake preparation process. The fracture process of the heterogeneous material surrounding the backbone of asperities distributed along the activated fault undergoes a phase transition phenomenon similar to one that the human heart undergoes during its transition from the healthy state to the pathological one. When the fracture of heterogeneous medium is completed, the stresses siege the backbone of strong entities distributed along the fault preventing the relative slip of its two faces (Contoyiannis et al., 2004, 2005). The observed $\mathrm{kHz}$ EM anomalies signalize the fracture of the aforementioned backbone. In a recent work we have shown, by means of nonextensive statistical mechanics, the existence of a dynamical analogy between a single fault activation in terms of $\mathrm{kHz} \mathrm{EM}$ activity/a single seizure activation. Namely, a dynamical analogy exists for the ways in which firing neurons/opening cracks organize themselves to produce a single epileptic seizure/EQ (Li et al., 2005; Kapiris et al., 2005; Eftaxias et al., 2006, 2012). We have also found that the dynamics of magnetic storms, solar flares, earthquakes and preseismic $\mathrm{kHz}$ EM anomalies also show dynamical analogies (Balasis et al., 2011a, b). In summary, the evolution of earthquake preparation process is accompanied by appearance of a heart-failure-type "disease", which is followed by the emergence of an epileptic-seizure-type crisis.

A corollary in the study of complex systems is that by transferring well-documented ideas and results from investigations in hitherto disparate areas we can check our proposals and results. This procedure is very useful especially in the study of EM precursors considering the difficulties associated with factors such as their highly complex nature, the rarity of large EQs and subtleties of possible clear EM pre-seismic signatures, and the present negative views in literature concerning their existence. The present work extends our proposals concerning the existence of common pathological symptoms in preseismic EM emission and other different well-studied "pathological" events further supporting the precursory nature of the observed EM anomalies.

Acknowledgements. Research co-funded by the EU (European Social Fund) and national funds, action "Archimedes III-Funding of research groups in T.E.I.", under the Operational Programme "Education and Lifelong Learning 2007-2013".

Edited by: M. E. Contadakis

Reviewed by: K. A. Chishko and one anonymous referee

\section{References}

Abe, S. and Suzuki, N.: Statistical similarities between internetquakes and earthquakes, Physica D, 193, 310-314, 2004.

Balasis, G., Daglis, I. A., Anastasiadis, A., Papadimitriou, C., Mandea, M., and Eftaxias, K.: Universality in solar flare, magnetic storm and earthquake dynamics using Tsallis statistical mechanics, Physica A, 390, 341-346, 2011a.

Balasis, G., Papadimitriou, C., Daglis, I. A., Anastasiadis, A., Sandberg, I., and Eftaxias, K.: Similarities between extreme events in the solar-terrestrial system by means of nonextensivity, Nonlin. Processes Geophys., 18, 563-572, doi:10.5194/npg-18-5632011, $2011 b$.

Bar-Yam, Y.: Dynamics of complex systems, Addison-Wesley, Reading, Massachusetts, 1997.

Boudjada, M. Y., Schwingenschuh, K., Döller, R., Rohznoi, A., Parrot, M., Biagi, P. F., Galopeau, P. H. M., Solovieva, M., Molchanov, O., Biernat, H. K., Stangl, G., Lammer, H., Moldovan, I., Voller, W., and Ampferer, M.: Decrease of VLF transmitter signal and Chorus-whistler waves before 1'Aquila earthquake occurrence, Nat. Hazards Earth Syst. Sci., 10, 14871494, doi:10.5194/nhess-10-1487-2010, 2010.

Bousseljot, R., Kreiseler, D., and Schnabel, A.: Nutzung der EKG-Signaldatenbank CARDIODAT der PTB über das Internet, Biomedical Engineering/Biomedizinische Technik, 40, 317-318, 1995.

Bowman, D., Quillon, G., Sammis, C., Sornette, A., and Sornette, D.: An observational test of the critical earthquake concept, J. Geophys. Res., 103, 24359-24372, 1998.

Contoyiannis, Y. F. and Diakonos, F. K.: Unimodal maps and order parameter fluctuations in the critical region, Phys. Rev. E, 76, 031138, doi:10.1103/PhysRevE.76.031138, 2007.

Contoyiannis, Y. F. and Eftaxias, K.: Tsallis and Levy statistics in the preparation of an earthquake, Nonlin. Processes Geophys., 15, 379-388, doi:10.5194/npg-15-379-2008, 2008.

Contoyiannis, Y. F., Diakonos, F. K., and Malakis, A.: Intermittent Dynamics of Critical Fluctuations, Phys. Rev. Lett., 89, 035701, doi:10.1103/PhysRevLett.89.035701, 2002.

Contoyiannis, Y. F., Diakonos, F. K., Papaefthimiou, C., and Theophilidis, G.: Criticality in the Relaxation Phase of a Spontaneously Contracting Atria Isolated from a Frog's Heart, Phys. Rev. Lett., 93, 098101, doi:10.1103/PhysRevLett.93.098101, 2004.

Contoyiannis, Y. F., Kapiris, P., and Eftaxias, C.: Monitoring of a preseismic phase from its electromagnetic precursors, Phys. Rev. E, 71, 066123, doi:10.1103/PhysRevE.71.066123, 2005. 
Contoyiannis, Y. F., Nomicos, C., Kopanas, J., Antonopoulos, G., Contoyianni, L., and Eftaxias, K.: Critical features in electromagnetic anomalies detected prior to the L'Aquila earthquake, Physica A, 389, 499-508, 2010.

Daubechies, I.: Ten lectures on wavelets, 2nd Edn., SIAM, Society for Industrial and Applied Mathematics, 1992.

de Arcangelis, L., Godano, C., Lippiello, E., and Nicodemi, M.: Universality in solar flare and earthquake occurrence, Phys. Rev. Lett., 96, 051102, doi:10.1103/PhysRevLett.96.051102, 2006.

Di Marco, L. Y., Duan, W., Bojarnejad, M., Zheng, D., King, S., Murray, A., and Langley, P.: Evaluation of an algorithm based on single-condition decision rules for binary classification of 12-lead ambulatory ECG recording quality, Physiol. Meas., 33, 1435-1448, 2012.

Diakonos, F. K., Pingel, D., and Schmelcher, P.: A stochastic approach to the construction of fully chaotic maps with prescribed statistical properties, Phys. Lett. A, 264, 162-170, 1999.

Eftaxias, K., Kapiris, P., Polygiannakis, J., Bogris, N., Kopanas, J., Antonopoulos, G., Peratzakis, A., and Hadjicontis, V.: Signature of pending earthquake from electromagnetic anomalies, Geophys. Res. Lett., 28, 3321-3324, 2001.

Eftaxias, K., Frangos, P., Kapiris, P., Polygiannakis, J., Kopanas, J., Peratzakis, A., Skountzos, P., and Jaggard, D.: Review-Model of Pre-Seismic Electromagnetic Emissions in Terms of FractalElectrodynamics, Fractals, 12, 243-273, 2004.

Eftaxias, K. A., Kapiris, P. G., Balasis, G. T., Peratzakis, A., Karamanos, K., Kopanas, J., Antonopoulos, G., and Nomicos, K. D.: Unified approach to catastrophic events: from the normal state to geological or biological shock in terms of spectral fractal and nonlinear analysis, Nat. Hazards Earth Syst. Sci., 6, 205-228, doi:10.5194/nhess-6-205-2006, 2006.

Eftaxias, K., Athanasopoulou, L., Balasis, G., Kalimeri, M., Nikolopoulos, S., Contoyiannis, Y., Kopanas, J., Antonopoulos, G., and Nomicos, C.: Unfolding the procedure of characterizing recorded ultra low frequency, $\mathrm{kHZ}$ and $\mathrm{MHz}$ electromagetic anomalies prior to the L'Aquila earthquake as preseismic ones - Part 1, Nat. Hazards Earth Syst. Sci., 9, 19531971, doi:10.5194/nhess-9-1953-2009, 2009.

Eftaxias, K., Balasis, G., Contoyiannis, Y., Papadimitriou, C., Kalimeri, M., Athanasopoulou, L., Nikolopoulos, S., Kopanas, J., Antonopoulos, G., and Nomicos, C.: Unfolding the procedure of characterizing recorded ultra low frequency, $\mathrm{kHZ}$ and $\mathrm{MHz}$ electromagnetic anomalies prior to the L'Aquila earthquake as pre-seismic ones - Part 2, Nat. Hazards Earth Syst. Sci., 10, 275294, doi:10.5194/nhess-10-275-2010, 2010.

Eftaxias, K.: Are There Pre-Seismic Electromagnetic Precursors? A Multidisciplinary Approach, in: Earthquake Research and Analysis - Statistical Studies, Observations and Planning, Intech, online, ISBN: 978-953-51-0134-5, doi:10.5772/28069, 2011.

Eftaxias, K., Minadakis, G., Potirakis, S. M., and Balasis, G.: Dynamical analogy between epileptic seizures and seismogenic electromagnetic emissions by means of nonextensive statistical mechanics, Physica A, 392, 497-509, doi:10.1016/j.physa.2012.09.015, 2013

Fukuda, K., Nunes, L., and Stanley, H. E.: Similarities between communication dynamics in the Internet and the automatic nervous system, Europhys. Lett., 62, 189-195, 2003.

Goldberger, A. L., Amaral, L. A. N., Glass, L., Hausdorff, J. M., Ivanov, P. C., Mark, R. G., Mietus, J. E., Moody, G. B., Peng,
C.-K., and Stanley, H. E.: PhysioBank, PhysioToolkit, and PhysioNet: Components of a New Research Resource for Complex Physiologic Signals, Circulation, 101, e215-e220, 2000.

Goldberger, A. L., Amaral, L. A. N., Hausdorff, J. M., Ivanov, P. C., Peng, C.-K., and Stanley, H. E.: Fractal dynamics in physiology: Alterations with disease and aging, Proc. Natl. Aca. Sci., 99, 2466-2472, 2002.

Heneghan, C. and McDarby, G.: Establishing the relation between detrended fluctuation analysis and power spectral density analysis for stochastic processes, Phys. Rev. E, 62, 6103-6110, 2000.

Herz, A. and Hopfield, J.: Earthquake cycles and neural reverberations: collective oscillations in systems with pulse-coupled threshold elements, Phys. Rev. Lett., 75, 1222-1225, 1995.

Hopfield, J.: Neurons, dynamics and computation, Physics Today, 40, 40-46, 1994.

Hurst, H.: Long term storage capacity of reservoirs, Trans. Am. Soc. Civ. Eng., 116, 770-808, 1951.

Ivanov, P. C., Amaral, L. A. N., Goldberger,A. L., Havlin, S., Rosenblum, M. G., Struzik, Z. R., and Stanley, H. E.: Multifractality in human heartbeat dynamics, Nature, 399, 461-465, 1999.

Jaggard, D.: On fractal electrodynamics, in: Recent Advances in Electromagnetic Theory, edited by: Kritikos, H. and Jaggard, D., Springer-Verlag, New York, 183-224, 1990.

Kapiris, P. G., Eftaxias, K. A., Nomikos, K. D., Polygiannakis, J., Dologlou, E., Balasis, G. T., Bogris, N. G., Peratzakis, A. S., and Hadjicontis, V. E.: Evolving towards a critical point: A possible electromagnetic way in which the critical regime is reached as the rupture approaches, Nonlin. Processes Geophys., 10, 511524, doi:10.5194/npg-10-511-2003, 2003.

Kapiris, P. G., Eftaxias, K. A., and Chelidze, T. L.: Electromagnetic signature of prefracture criticality in heterogeneous media, Phys. Rev. Lett., 92, 065702, doi:10.1103/PhysRevLett.92.065702, 2004.

Kapiris, P., Polygiannakis, J., Yao, X., and Eftaxias, K.: Similarities in precursory features in seismic shocks and epileptic seizures, Europhys. Lett., 69, 657-663, 2005.

Karamanos, K., Dakopoulos, D., Aloupis, K., Peratzakis, A., Athanasopoulou, L., Nikolopoulos, S., Kapiris, P., and Eftaxias, K.: Preseismic electromagnetic signals in terms of complexity, Phys. Rev. E, 74, 016104, doi:10.1103/PhysRevE.74.016104, 2006.

Kim, Y. and Kim, E.: Effectiveness of the continuous wavelet transform in the analysis of some dispersive elastic waves, J. Acoust. Soc. Am., 110, 86-94, 2001.

Kiyono, K., Struzik, Z. R., Aoyagi, N., Sakata, S., Hayano, J., and Yamamoto, Y.: Critical Scale Invariance in a Healthy Human Heart Rate, Phys. Rev. Lett., 93, 178103, doi:10.1103/PhysRevLett.93.178103, 2004.

Kiyono, K., Struzik, Z. R., Aoyagi, N., Togo, F., and Yamamoto, Y.: Phase Transition in a Healthy Human Heart Rate, Phys. Rev. Lett., 95, 058101, doi:10.1103/PhysRevLett.95.058101, 2005.

Kossobokov, V., Keillis-Borok, V., and Cheng, B.: Similarities of multiple fracturing on a neutron star and on Earth, Phys. Rev. E, 61, 3529-3533, 2000.

Kreiseler, D. and Bousseljot, R.: Automatisierte EKG-Auswertung mit Hilfe der EKG-Signaldatenbank CARDIODAT der PTB, Biomedical Engineering/Biomedizinische Technik, 40, 319-320, 1995. 
Lacidogna, G., Carpinteri, A., Manuello, A., Durin, G., Schiavi, A., Niccolini, G., and Agosto, A.: Acoustic and electromagnetic emissions as precursors phenomena in failure processes, Strain, 47 (Suppl. 2), 144-152, 2011.

Li, C.-F. and Liner, C.: Singularity exponent from wavelet-based multiscale analysis: A new seismic attribute, Chin. J.Geoph., 48, 953-959, 2005.

Li, X., Polygiannakis, J., Kapiris, P., Peratzakis, A., Eftaxias, K., and Yao, X.: Fractal spectral analysis of pre-epileptic seizures in terms of criticality, J. Neur. Eng., 2, 1-6, 2005.

Lin, D. C. and Hughson, R. L.: Modeling Heart Rate Variability in Healthy Humans: A Turbulence Analogy, Phys. Rev. Lett, 86, 1650-1653, 2001.

Lowen, S. B. and Teich, M. C.: Estimation and simulation of fractal stochastic point processes, Fractals, 3, 183-210, 1995.

Mandelbrot, B. B. and Wallis, J. R.: Noah, Joseph and operational hydrology, Water Resour. Res., 4, 909-918, 1968.

Mallat, S.: A wavelet tour of signal processing, Academic Press, 1998.

Mallat, S. and Hwang, W.-L.: Singularity detection and processing with wavelets, Trans. Inf. Theory, 38, 617-642, 1992.

Minadakis, G., Potirakis, S. M., Nomicos, C., and Eftaxias, K.: Linking electromagnetic precursors with earthquake dynamics: an approach based on nonextensive fragment and self-affine asperity models, Physica A, 391, 2232-2244, 2012.

Nawab, S. H. and Quatieri, T.: Short-time Fourier transform, in: Advanced Topics in Signal Processing, edited by: Lim, J. S. and Oppenheim, A. V., Englewood Cliffs, NJ: Prentice-Hall, 1988.

Oppenheim, A. V., Schafer, R. W., and Buck, J. R.: Discrete-time signal processing, 2nd Edn., Prentice-Hall, Inc., Upper Saddle River, NJ, USA, 1999.

Osorio, I., Frei, M. G., Sornette, D., Milton, J., and Lai, Y.-C.: Epileptic seizures: Quakes of the brain?, Phys. Rev. E, 82, 021919, doi:10.1103/PhysRevE.82.021919, 2010.

Papadimitriou, C., Kalimeri, M., and Eftaxias, K.: Nonextensivity and universality in the EQ preparation process, Phys. Rev. E, 77, 036101, doi:10.1103/PhysRevE.77.036101, 2008.

Pergola, N., Aliano, C., Coviello, I., Filizzola, C., Genzano, N., Lacava, T., Lisi, M., Mazzeo, G., and Tramutoli, V.: Using RST approach and EOS-MODIS radiances for monitoring seismically active regions: a study on the 6 April 2009 Abruzzo earthquake, Nat. Hazards Earth Syst. Sci., 10, 239-249, doi:10.5194/nhess10-239-2010, 2010.

Peters, O., Hertlein, C., and Christensen, K.: A complexity view of rainfall, Phys. Rev. Lett., 88, 018701, doi:10.1103/PhysRevLett.88.018701, 2002.

Picoli, S., Mendes, R., Malacarne, L., and Papa, A.: Similarities between the dynamics of geomagnetic signal and heartbeat intervals, Europhys. Lett., 80, 50006, doi:10.1209/02955075/80/50006, 2007.

Pingel, D., Schmelcher, P., and Diakonos, F. K.: Theory and examples to the inverse Frobenius-Perron problem for complete chaotic maps, Chaos, 9, 357, doi:10.1063/1.166413, 1999.

Pinsky, M.: Introduction to Fourier Analysis and Wavelets, Brooks/Cole, ISBN 0-534-37660-6, 2002.
Potirakis, S. M., Minadakis, G., and Eftaxias, K.: Analysis of electromagnetic pre-seismic emissions using Fisher information and Tsallis entropy, Physica A, 391, 300-306, doi:10.1016/j.physa.2011.08.003, 2012a.

Potirakis, S. M., Minadakis, G., and Eftaxias, K.: Relation between seismicity and pre-earthquake electromagnetic emissions in terms of energy, information and entropy content, Nat. Hazards Earth Syst. Sci., 12, 1179-1183, doi:10.5194/nhess-121179-2012, 2012b.

Potirakis, S. M., Minadakis, G., and Eftaxias, K.: Sudden drop of fractal dimension of electromagnetic emissions recorded prior to significant earthquake, Nat. Hazards, 64, 641-650, doi:10.1007/s11069-012-0262-x, 2012c.

Rioul, O. and Duhamel, P.: Fast Algorithms for Discrete and Continuous Wavelet Transforms, Trans. Inf. Theory, 38, 569-586, 1992.

Rundle, J. B., Tiampo, K. F., Klein, W., and Sa Martins, J. S.: Selforganization in leaky threshold systems: the influence of nearmean field dynamics and its implications for EQs, neurology, and forecasting, Proc. Natl. Aca. Sci., 99, 2514-2521, 2002.

Schuster, H. G.: Deterministic chaos, VCH, Weinheim, 1998.

Sornette, D. and Helmstetter, A.: Occurrence of finitetime singularities in epidemic models of rupture, earthquakes and starquakes, Phys. Rev. Lett., 89, 158501, doi:10.1103/PhysRevLett.89.158501, 2002.

Stanley, H. E.: Introduction to Phase Transitions and Critical Phenomena, Oxford University Press, 1987.

Stanley, H. E.: Scaling, universality, and renormalization: Three pillars of modern critical phenomena, Rev. Modern Phys., 71, S358-S366, 1999.

Stark, H.-G.: Wavelets and Signal Processing, Springer-Verlag, Berlin, Heidelberg, New York, ISBN 3-540-23433-0, 2005.

Torrence, C. and Compo G. P.: A practical guide to wavelet analysis, Bull. Am. Meteorol. Soc., 79, 61-78, 1998.

Varotsos, P., Sarlis, N., Scordas, E., and Lazaridou, M.: Identifying sudden cardiac death risk and specifying its occurrence time by analyzing electrocardiograms in natural time, Appl. Phys. Lett., 91, 064106, doi:10.1063/1.2768928, 2007.

Varotsos, P., Sarlis, N. V., and Skordas, E. S.: Natural Time Analysis: The New View of Time, Berlin, Springer, 2011a.

Varotsos, P., Sarlis, N. V., Skordas, E. S., Uyeda, S., and Kamogawa, M.: Natural time analysis of critical phenomena, Proc. Natl. Aca. Sci., 108, 11361, doi:10.1073/pnas.1108138108, $2011 \mathrm{~b}$.

Walters, R., Elliot, J., D Agostino, N., England, P., Hunstad, I., Jackson, J., Parsons, B., Phillips, R., and Roberts, G.: The 2009 L'Aquila earthquake (central Italy): A source mechanism and implications for seismic hazard, Geophys. Res. Lett., 36, L17312, doi:10.1029/2009GL039337, 2009.

Weeks, M.: Digital Signal Processing Using MATLAB and Wavelets, Infinity Science Press, Hingham, MA, ISBN: 09778582-0-0, 2007.

Yeh, P.-L. and Liu, P.-L.: Application of the wavelet transform and the enhanced Fourier spectrum in the impact echo test, NDT\&E Internatl., 41, 382-394, 2008. 